\title{
Médiévales
}

Langues, Textes, Histoire

77 | automne 2019

Mathématiques. Savoirs et enseignements (OrientOccident)

Hanna Vorholt, Shaping Knowledge. The Transmission of the Liber FloridusLondres, Warburg Institute ("Warburg Institute Studies and Texts ", 6), 2017, 327 p. Nathalie Bouloux (Université de Tours, CESR)

\section{Albert Derolez, The Making and Meaning of the Liber Floridus : A Study of the Original Manuscript}

Ghent, University Library MS 92, Londres/Turnhout, Harvey Miller/ Brepols (« Studies in Medieval and Early Renaissance Art History », 76), 2015,355 p.

\section{Nathalie Bouloux}

\section{OpenEdition \\ Journals}

Édition électronique

URL : https://journals.openedition.org/medievales/10682

DOI : 10.4000/medievales. 10682

ISSN : 1777-5892

Éditeur

Presses universitaires de Vincennes

Édition imprimée

Date de publication : 5 décembre 2019

Pagination : 188-192

ISBN : 978-2-37924-061-4

ISSN : 0751-2708

\section{Référence électronique}

Nathalie Bouloux, «Albert Derolez, The Making and Meaning of the Liber Floridus : A Study of the Original Manuscript », Médiévales [En ligne], 77 | automne 2019, mis en ligne le 12 mai 2020, consulté le 23 avril 2022. URL : http://journals.openedition.org/medievales/10682 ; DOI : https://doi.org/ 10.4000/medievales.10682 
Albert Derolez, The Making and Meaning of the Liber Floridus : A Study of the Original Manuscript, Ghent, University Library MS 92, Londres/Turnhout, Harvey Miller/ Brepols (« Studies in Medieval and Early Renaissance Art History », 76), 2015, 355 p.

Hanna Vorholt, Shaping Knowledge. The Transmission of the Liber Floridus, Londres, Warburg Institute («Warburg Institute Studies and Texts », 6), 2017, 327 p.

Le livre d'Albert Derolez couronne cinquante ans de recherches sur celui qu'il désigne lui-même comme « un obscur chanoine flamand », Lambert de Saint-Omer (actif au début du XII ${ }^{\mathrm{e}}$ siècle), auteur du Liber Floridus. L'ouvrage porte sur le manuscrit autographe, dont la signification complexe ne peut être comprise que par l'examen attentif et minutieux de sa structure et de son contenu. Contrairement à ce que son titre pourrait faire entendre, le Liber Floridus n'est pas un florilège, mais une œuvre d'édification construite sur la compilation d'un grand nombre de sources sélectionnées, organisées et adaptées selon les vues de Lambert, augmentée de chapitres originaux et illustrée d'images, de tables, de cartes, de diagrammes et de tables qui en font une œuvre remarquable.

Le livre s'ouvre sur une section rassemblant d'une part des sources utilisées par Lambert, d'autre part une bibliographie critique. L'ensemble se caractérise par une volonté de clarté, qui vient de la conscience d'A. Derolez des difficultés à rendre compte à la fois de la composition du manuscrit et des retouches incessantes qu'y a portées Lambert, brouillant ainsi les pistes relatives à l'élaboration et à la signification de son œuvre.

L'introduction rappelle les principaux apports de l'historiographie récente, depuis le travail inaugural de Léopold Delisle paru en 1906. Elle présente le peu que l'on sait de Lambert et le manuscrit autographe du Liber Floridus, élaboré entre 1006-1111 et juin 1121 (parchemin, structure des cahiers, composition, écriture, et enluminures). Elle s'achève par une présentation générale du texte et de la table des matières, et sur la question des sources utilisées par Lambert. À la suite, A. Derolez guide le lecteur dans le manuscrit, folio par folio : les textes préliminaires (chapitre 1) ; la partie centrale (chapitre 2) ; les suppléments (chapitres 3 à 6); les derniers chapitres (chapitres 7 et 8). Le chapitre 9 résume l'ensemble des données pour livrer une vision synthétique de l'élaboration du Liber Floridus en treize phases. En conclusion, A. Derolez rassemble ses principaux résultats et donne son interprétation des buts de Lambert et de la signification de son œuvre. Une série d'appendices conclut le livre : I. Les diverses copies du Liber Floridus ; II. Les manuscrits ayant servi de sources au Liber Floridus. La fin du livre reproduit les principales illustrations du livre (120), soit d'après le manuscrit autographe, soit d'après d'autres manuscrits lorsqu'elles ont disparu du premier. Trois tables constituent des instruments de travail indispensables pour comprendre la manière de procéder d'A. Derolez : une première présente la structure actuelle du manuscrit ; la deuxième la genèse des cahiers en vue de retracer les modifications apportées par Lambert ; la troisième regroupe ceux qui présentent une réglure similaire (et sont donc de fabrication proche); la dernière donne la structure de la table des matières, dressée par Lambert lui-même à un moment où il considérait comme achevé un ouvrage qu'il allait pourtant retoucher, si bien que la table ne correspond pas tout à fait au contenu actuel du Liber Floridus, d'autant que certains cahiers ont disparu - notamment ceux consacrés à l'Apocalypse.

Ces instruments de travail sont indispensables pour comprendre la méthode et le but d'A. Derolez, qui vise à reconstituer pas à pas les étapes de la rédaction du livre, en tenant compte des remaniements constants opérés par Lambert de Saint-Omer. En une vingtaine d'années, celui-ci a ajouté, déplacé des folios, gratté ou réécrit des textes, en vue d'adapter le livre qu'il fabriquait à l'évolution de sa pensée ou à la découverte de nouvelles sources. Le titre du livre correspond donc parfaitement à son objet, et malgré une abondance de détails étudiés avec minutie, on suit avec intérêt son analyse qui démontre le caractère indispensable de la codicologie pour étudier les œuvres et la pensée des auteurs 
médiévaux. C'est une réussite. Le seul bémol peut-être est le recours trop dispendieux qu'A. Derolez fait des instruments de travail réunis en fin de volume, pourtant une aide indispensable à la lecture.

Lambert de Saint-Omer, chanoine de la cathédrale Notre-Dame, vit dans un environnement intellectuel relativement limité. Il fabrique son manuscrit sans bénéficier d'un vrai scriptorium, sans avoir toujours à disposition le parchemin nécessaire, ce qui le contraint à bricoler en collant, découpant, réutilisant et déplaçant des folios dès lors qu'il entend remanier son œuvre. Sa formation ne lui a pas permis de maîtriser parfaitement le latin. Les historiens ont souvent insisté sur les relations conflictuelles entre la cathédrale Notre-Dame de Saint-Omer et l'ancienne abbaye de Saint-Bertin, ce qui aurait interdit à Lambert de fréquenter assidûment la bibliothèque monastique. A. Derolez démontre cependant la correspondance entre des manuscrits conservés dans cette bibliothèque et les textes conservés dans le Liber Floridus, de même que les rapports étroits entre l'œuvre de Lambert et de textes historiques écrits par un moine de Saint-Bertin (London, British Library, Cotton Fragments 1). Il ne faut donc pas exagérer les difficultés de Lambert à fréquenter la bibliothèque du monastère. En revanche, il est exclu de faire de Lambert un savant de la Renaissance du XII ${ }^{\mathrm{e}}$ siècle. Son environnement intellectuel immédiat devait se limiter aux moines de Saint-Bertin et à la rencontre épisodique avec un grand intellectuel, Anselme de Canterbury. Il a pu connaître les travaux de Gilbert Crispin, d'Odon de Cambrai, de Hildebert du Mans et de Marbode de Rennes, mais certainement pas les ouvrages de Hugues de Saint-Victor ou de Honorius d'Autun. Ses sources principales sont des textes de l'Antiquité tardive et du haut Moyen Âge. Sa perspective est fondamentalement régionale et c'est à une interprétation eschatologique du destin des hommes et du monde vue depuis la Flandre qu'est consacré le Liber Floridus. L'ouvrage a pu parfois apparaître comme une médiocre compilation, dans la mesure où Lambert se contente de juxtaposer les extraits d'auteurs, mais l'étude attentive de la mise en forme du manuscrit révèle l'existence d'un projet préliminaire cohérent, mais sans doute insatisfaisant aux yeux de son auteur qui retravaille dès lors l'ordonnancement des folios, ce qui rend le cheminement de sa pensée difficile à saisir. C'est une autre des qualités d'A. Derolez que de rester prudent dans ses interprétations, toujours fondées sur l'examen attentif du manuscrit. Cette méthode permet de faire émerger les thèmes fondamentaux d'une œuvre consacrée au rôle des hommes dans le dessein de Dieu : la cosmologie, la cosmographie, le temps, la croisade, la place des juifs, l'histoire des régions nord-septentrionales de l'Europe (Angleterre, Normandie, Flandre)... Ce livre procure ainsi un point de départ indispensable à l'étude de l'œuvre de Lambert. A. Derolez s'y est essentiellement consacré au manuscrit autographe de Lambert et ne tient compte que ponctuellement des autres manuscrits, notamment celui conservé à Wolfenbüttel (voir ci-dessous), copie la plus proche du manuscrit de Gand qui permet d'en reconstituer les cahiers manquants.

La perspective du livre d'Hanna Vorholt est tout autre et parfaitement complémentaire, puisque l'auteure examine l'ensemble de la tradition manuscrite afin de comprendre la manière dont les copistes ont compris, copié et transformé l'œuvre de Lambert. Sa méthode repose sur l'idée de l'unicité de chaque entreprise de copie et de transfert du Liber Floridus, et le constat que chaque manuscrit, par la mise en page, la sélection des textes du Liber et l'ajout de nouveaux éléments, correspond à une transformation consciente du savoir mis en œuvre par Lambert. Chaque manuscrit vaut d'être étudié en lui-même, non comme témoin plus ou moins proche de l'œuvre d'origine, mais en tant qu'objet culturel dont le contexte d'élaboration et les interventions du copiste sont révélateurs de l'époque et du milieu où il a été conçu. 
L'auteure suit un plan chronologique et systématique. Elle traite successivement la réception du livre au XII ${ }^{\mathrm{e}}$, puis aux XIII ${ }^{\mathrm{e}}$ et $\mathrm{XIV}^{\mathrm{e}}$ siècles, enfin au XV ${ }^{\mathrm{e}}$ siècle. Chaque chapitre est construit sur une présentation des manuscrits du Liber Floridus produits à la période concernée puis par l'étude approfondie d'un manuscrit particulièrement significatif : pour le XII ${ }^{\mathrm{e}}$ siècle, le manuscrit de Wolfenbüttel (Wolfenbüttel, Herzog August Bibliothek, Cod. Guelf. 1 Gud. lat.) ; pour les XIII et XIV siècles, celui de Leyde (Leiden, Bibliotheek der Rijksuniversiteit, Voss. Lat. Fol. 31) ; et pour le $\mathrm{XV}^{\mathrm{e}}$ siècle, celui de Tongerlo (Tongerlo, Abdijarchief, Sectie V,Nr. 325). À la fin du volume, trois annexes parachèvent l'ensemble, la première consacrée à la description et à l'historique de conservation des trois manuscrits qui ont servi de base à l'étude ; une deuxième à une vue d'ensemble du contenu de chacun d'entre eux ; une troisième à la transmission des images.

L'auteure développe dans l'ensemble du livre des vues originales et bien argumentées, qui ouvrent d'incontestables perspectives dans le domaine des études de la réception d'une œuvre. L'introduction (ou premier chapitre) s'ouvre sur une synthèse historiographique des études consacrées à Lambert de Saint-Omer. L'auteure s'attache ensuite à définir les traits caractéristiques du Liber Floridus en montrant notamment que Lambert travaille principalement sur la base d'une pensée associative, ce qui est perceptible aussi bien dans les textes et leur organisation dans le manuscrit que dans les diagrammes.

Le chapitre II est principalement consacré au manuscrit de Wolfenbüttel qui, bien qu'incomplet, est à la fois le plus proche du manuscrit autographe et le plus souvent copié, si bien qu'il n'a que très rarement été étudié pour lui-même. Or l'auteure démontre que, si le projet d'origine du copiste était bien de suivre son modèle, il est intervenu de plus en plus fréquemment, jusqu'à faire des omissions, des additions et des modifications qui transforment consciemment le Liber Floridus. Le copiste a notamment travaillé en adaptant l'aspect du livre aux standards en vigueur à la fin du XII ${ }^{\mathrm{e}}$ siècle, par exemple en transformant la mise en page, continue dans l'autographe, sur deux colonnes dans le Wolfenbüttel. Il a souvent modifié l'unité texte et figures, altérant la signification de l'œuvre de Lambert. Quelques interventions sur les images ont les mêmes conséquences. Par exemple, alors que Lambert tend à dessiner le pape comme un souverain temporel, le copiste choisit, par de subtiles transformations du dessin, d'en faire un souverain spirituel, se conformant ainsi à une conception du pape répandue à la fin du XII ${ }^{\mathrm{e}}$ siècle. Le copiste a omis des données, comme les informations personnelles introduites par Lambert, une partie des Gesta Francorum, la carte de l'Europe, etc. Il a parfois corrigé les textes copiés par Lambert sur la base d'une autre version. Enfin, il a complété la généalogie des comtes de Flandres et, surtout, ajouté un traité néoplatonicien anonyme, le De anima mundi et concordia planetarum, accompagné d'un diagramme. À la suite de ce traité, il copie les passages anti-juifs du Liber Floridus qu'il combine avec des éléments qui lui sont étrangers, comme les Dialogues Melchi, des passages de Pline et du De differentia. De la sorte, le scribe et ses collaborateurs ont transformé l'œuvre de Lambert en corrigeant les textes, réorganisant certains passages et en insérant de nouveaux traités.

$\mathrm{Au} \mathrm{XIII}^{\mathrm{e}}$ et au XIV ${ }^{\mathrm{e}}$ siècle, le manuscrit autographe du Liber Floridus est conservé à l'abbaye bénédictine de Saint-Bavon à Gand où des compléments ponctuels sont apportés (par exemple, la bataille de Golden Spurs près de Courtrai en 1302 est ajoutée au calendrier). Un des exemplaires les plus intéressants de l'œuvre est celui de Leyde, copié sur le manuscrit de Wolfenbüttel et constitué de deux parties, Leyde A et Leyde B. Dans Leyde A, réalisé en trois phases, ont été ajoutées deux séries d'additions. La première traite de questions ecclésiastiques en relation avec la papauté et la politique de l'Église à la fin du XIII ${ }^{\mathrm{e}}$ siècle. La seconde rassemble le Proverbia rusticorum, les Sentences de Publius Syrus, des poèmes, et une oratio de Paulin de Nole. Dans la dernière partie du manuscrit (Leyde B), d'autres textes encore ont été ajoutés, parmi lesquels une satire 
antiféministe (De coniuge non ducenda), un traité de médecine, le Tacuinum corporum (dans la traduction commandée par Charles d'Anjou, roi de Naples, au physicien juif Faraj ben Solomon), le Compendium historiae in genealogia Christi de Pierre de Poitiers, suivi des Constitutions promulguées par le pape Grégoire x. Les images du manuscrit de Wolfenbüttel qui n'ont pas été copiées dans la première partie du manuscrit (Leyde A) l'ont été dans la seconde partie (Leyde B), ce qui est prouvé par la correspondance entre ces images et les marques laissées dans le manuscrit de Wolfenbüttel pour indiquer au copiste les images à insérer dans cette partie B. Les textes et les lettres ajoutés au manuscrit suggèrent qu'il a été copié pour une personnalité proche de la cour de France. C'est un bel exemple d'une reconstitution soignée du Liber Floridus augmentée d'un grand nombre d'additions.

$\mathrm{Au} \mathrm{Xv}^{\mathrm{e}}$ siècle, le Liber Floridus continue d'être copié principalement à partir du manuscrit de Wolfenbüttel, pour des commanditaires de haut rang, ce qui se manifeste dans les images, qui sont confiées aux meilleurs artistes. Philippe de Clèves (14561528 ) en fait faire une traduction, le Livre fleurissant en fleurs (Hagues, 128 C4). Mais le manuscrit conservé à Tongerlo est certainement un des plus remarquables par les modifications d'ampleur qu'il apporte au texte. Copié dans le dernier quart du Xv siècle à Louvain, à partir du manuscrit conservé aujourd'hui à Gênes et issu du Wolfenbüttel, il manifeste un intérêt particulier pour les images et les diagrammes, qui subissent souvent des transformations significatives. Dans ce manuscrit de grande taille, le texte du Liber Floridus occupe les folios 25 à 42 . Le travail de copie a donné lieu à un agencement original entre les textes et les images. Des textes et des diagrammes nouveaux apparaissent. Ainsi, un diagramme, illustrant la Genèse 1,9 , est issu des additions faites au commentaire de Nicolas de Lyre par Paul de Burgos (m. 1435). Il confronte la séparation des eaux et de la Terre à la théorie aristotélicienne des quatre éléments. De nouveaux textes sont ajoutés, tous en relation avec l'influence céleste sur la sphère terrestre. Les premiers cahiers du manuscrit, qui précèdent le Liber Floridus, font clairement échos à ce dernier. On y trouve des diagrammes composés en partie par Heymericus de Campo (m. 1460), professeur de théologie à Louvain et auteur d'un De sigillo eternitatis écrit au concile de Bâle entre 1432 et 1435. Ces diagrammes illustrent la mise en relation du cosmos avec la Terre en vue de connaître le divin par la contemplation visuelle de son œuvre. À la suite, d'autres textes sont insérés suivant un ordre chronologique (les six jours de la création, le Compendium historiae de Pierre de Poitiers où se trouvent d'autres diagrammes, dont un représentant Jérusalem, une version augmentée du Chronicon pontificum et imperatorum de Martin de Troppau). Ce manuscrit, centré sur le Liber Floridus, est un témoin remarquable d'une réception active du texte, orientée en particulier sur la relation texte/image dans la page manuscrite et la composition de diagrammes qui s'éclairent mutuellement tout au long du manuscrit.

H. Vorholt a admirablement démontré combien la nature même du Liber Floridus, fondé sur une logique associative, l'utilisation des rapports textes/images et la mise en relation des diverses parties du texte par des associations visuelles où les diagrammes jouent un rôle essentiel, a abouti à une réception très particulière de l'œuvre. Le Liber Floridus a inspiré aux concepteurs des manuscrits le désir de transformer la signification du livre par des ajouts et de subtiles transformations. Dans ce travail remarquable, un aspect aurait toutefois mérité d'être discuté. Au début du XII siècle, Lambert travaille selon une conception de l'univers caractéristique de l'Antiquité tardive et des temps carolingiens, où le cosmos est ordonné par la succession des sept ou huit sphères célestes qui entourent la Terre. À partir du XII ${ }^{\mathrm{e}}$ siècle et plus encore du XIII ${ }^{\mathrm{e}}$ siècle, la diffusion du modèle aristotélicien de l'univers transforme la conception du cosmos, caractérisée par la stricte division entre monde sublunaire et monde supralunaire, et christianisée notamment 
par l'introduction du ciel aqueux et de l'empyrée, demeure de Dieu, des anges et des élus. Les concepteurs des manuscrits en sont alors imprégnés et une part importante de leur travail d'interprétation du cosmos tel que décrit par Lambert consiste en une adaptation aristotélicienne du contenu du Liber Floridus.

Ces deux ouvrages sont remarquables : le premier, qui dresse le bilan de l'élaboration de l'œuvre telle qu'elle est conservée dans le manuscrit autographe, le second qui étudie avec minutie les stratégies des copistes pour transformer et moderniser le traité de Lambert. Indispensables pour tous ceux qui s'intéresseront au Liber Floridus, ils montrent aussi combien l'intérêt porté à la matérialité de l'écrit permet d'approfondir notre compréhension des cultures médiévales.

Nathalie Bouloux (Université de Tours, CESR)

Catherine VERNA, L'Industrie au village. Essai de microhistoire (Arles-sur-Tech, XIV et $X V^{\mathcal{e}}$ siècles), Paris, Les Belles Lettres (« Histoire », 140), 2017, $560 \mathrm{p}$.

Catherine Verna nous propose dans cet ouvrage dense et fouillé, non seulement une belle enquête sur une vallée des Pyrénées catalanes (la vallée du Tech) peu connue des chercheurs, mais surtout une remarquable réflexion méthodologique sur l'étude de l'essor industriel - essentiellement métallurgique - durant les derniers siècles du Moyen Âge. Les six parties réunissant les treize chapitres qui composent l'ouvrage se présentent comme autant de moyens d'aborder quelques-unes des multiples facettes du phénomène aux $\mathrm{XIV}^{\mathrm{e}}$ et $\mathrm{XV}^{\mathrm{e}}$ siècles et les observations recueillies dans les très nombreuses sources archivistiques sont confrontées aux problématiques et discussions historiographiques les plus récentes. Ce mode de faire est manifeste dès l'introduction, puisque l'auteure souligne, en balayant une large bibliographie, combien les historiens ont été rétifs à concevoir l'existence même d'une forme d'industrie à la fin du Moyen Âge et combien des notions essentielles comme celle de Verlagssystem ou de proto-industrie-étroitement associées à la prédominance d'un centre décisionnel urbain - peinent à rendre compte de la réalité complexe d'un mode de production rural antérieur à l'époque moderne. C'est pourquoi elle plaide pour distinguer ce moment singulier où les hommes des campagnes ont su développer, dans leurs ateliers, des activités qui permettaient à la fois une production artisanale et une production industrielle - au sens de production de masse comme l'a suggéré Philippe Braunstein.

Elle se propose de le démontrer en analysant les données archivistiques provenant d'amples dépouillements - plusieurs milliers d'actes notariés, des registres de la procuration royale conservés à Perpignan ou du Real Patrimonio à Barcelone - concernant le bourg d'Arles-sur-Tech et, dans une moindre mesure, ceux de Cérêt et de Prats-deMollo. Ces agglomérations comptaient parmi les plus importantes du Vallespir, une vallée pyrénéenne qui semblait prospère avant la guerre catalano-aragonaise (1462-1472). Ces bourgs se singularisaient des autres agglomérations de la vallée par l'ampleur des services qu'ils proposaient : ils étaient des centres administratifs, des lieux de marchés ; ils accueillaient des artisans spécialisés, des médecins, des apothicaires et des notaires.

C. Verna s'étend longuement sur le rôle des notaires. Ils étaient une quinzaine entre 1300 et 1450 à Arles-sur-Tech, largement investis dans le service de l'administration royale et les activités artisanales ou industrielles locales. Ils étaient insérés dans les nombreux réseaux de sociabilité (confréries, parentés, etc.) qui unissaient certains des hommes les plus puissants et les plus actifs de la vallée. Les actes qu'ils ont produits portent traces de savoirs techniques pointus qu'ils ont pu connaître - notamment par l'emploi d'un lexique latin ou catalan particulier - et traduisent, à l'occasion, l'existence d'innovation. Le vocabulaire contribue ainsi à définir un territoire technique, où les 